\title{
Leadership Convergence: An Application of Within and Between Analysis to Validity
}

\author{
Steven E.Marh

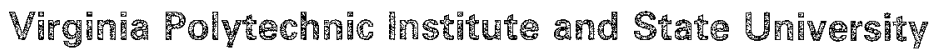 \\ Tred Damsareau, din. and Joseph A. Alutho \\ State University of Mew Yorle at Buffralo \\ MacDonald Dumas

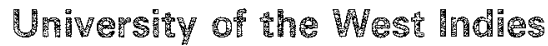

\begin{abstract}
Problems in drawing inferences about leadership phenomena when multiple units of analysis (groups and individuals) simultaneously exist in a data set are addressed. Using a technique recommended by Dansereau and Dumas (1977), within-unit and betweenunit sources of covariation are examined for data con taining matched superior-subordinate reports. In this data set matched superior-subordinate reports were not significantly correlated at the individual level. When supervisory group differences were held constant, however, the relationships between these matched reports were significantly greater than zero. This convergent validity within supervisory units suggests an approach to validity which is not included in traditional theories of leadership.
\end{abstract}

In discussing data aggregation problems, Kimberly $(1980$, p. 367) asked, "How valid is it . . to define leadership style in terms of averaged subordinate ratings?" The methodological issue of aggregation as it applies to the validity of superior and subordinate descriptions of leadership is the focus of this study. The problem raised by this issue can be viewed as one variant of a more general unit-of-analysis problem in organizational research (see Dansereau \& Dumas, 1977; Knapp, 1977).

A unit-of-analysis problem occurs when a data set contains reports from or about individuals who

APPLIED PSYCHOLOGICAL MEASUREMENT

Vol. 7, No. 1, Winter 1983, pp. 63-72

(c) Copyright 1983 Applied Psychological Measurement Inc. 0146-6216/831010063-10\$1.50 are located in maturally occurring groups. Group averages can be computed, correlated, and compared in such a data set. The inferences which are drawn from these aggregated average scores can be problematic, as discussed by Robinson (1950) in his famous "ecological fallacy" article. Simply stated, correlations (or any statistic) based upon aggregated scores cannot be used to draw inferences about the behavior of the individuals represented in the aggregation. Thus, the statistical unit of analysis should match the unit specified by a theory.

Nevertheless, a multiple-unit data set with individuals identified as members of groups contains untapped research potential. Dansereau and Dumas (1977), Knapp (1977), and Sirotnik (1980), among others, suggested that a variety of statistics and inferential procedures which do not contradict Robinson's ecological fallacy argument can be derived from a data base which contains individuals embedded in their groups (or collectivities). The Covariance Theorem from which Robinson's (1950) argument originates illustrates this point. The theorem (cf. Przeworski \& Teune, 1970) can be stated as follows:

$$
\begin{aligned}
& r_{x y, N^{\sigma} x, N^{\sigma} y, N}=r_{x y, B} B_{x, B}^{\sigma} y, B \\
& +\sum_{W=1} r_{x y, W^{\sigma} x, W^{\sigma} y, W}
\end{aligned}
$$


where $N=$ total number of individuals,

$B=$ group effect,

$J=$ total number of groups, and

$W=$ within-group effect.

In other words, as shown in Equation 1, the total covariation between variable $x$ and variable $y$ with $N$ individuals embedded in $J$ groups is equal to a weighted sum of the within-group and betweengroup covariances (Przeworski \& Teune, 1970, p. 59). Thus, three types of correlations can be derived for this type of multiple-unit data base, which contains $N$ individuals and $J$ groups. These three correlations are (1) the total raw unadjusted correlation, which is based on the total $N$ individuals; (2) the between-unit correlation, which is based on $J$ units, with each unit represented by its average score; and (3) a within-unit correlation based on the residual variation after removing between-unit differences.

The Covariance Theorem has important implications for research in formal work organizations because such orgamizations can be represented by a multiple-unit data base. An illustration from the leadership literature may help demonstrate this unitof-analysis issue. Katerburg and Hom (1981) examined descriptions of leadership behaviors to determine whether between-supervisory group or within-supervisory group variance was more important. In this type of research, a common approach is to ask subordinates their degree of agreement with statements such as "My supervisor looks out for the personal welfare of the group members." To the extent that all members of a supervisory unit respond with similar answers, then the unit's average score could be used to represent that supervisor's leadership style with some degree of accuracy. If some supervisors in an organization have high average scores on scales constructed from questions like the one above, and if other supervisors have low scores, a between-unit phenomenon could be described by using group averages to represent each supervisor's style. In this case, a between-unit correlation, which forms the second part of the Covariance Theorem, might prove useful.

There is, however, no guarantee that a supervisor treats all the members of his/her unit in a similar manner. It is hypothetically possible that subordinate reports in the same group could vary so widely that the use of the group average to represent the supervisor's style would create "a fictitious average or middle range score which the leader never displays" (Schriesheim, House, \& Kerr, 1976 , p. 361). For this case, a within-unit residual correlation, which is part of the third term of the Covariance Theorem, might prove more useful than a correlation based on unit averages.

Without empirical testing it is difficult to determine if a within-unit correlation or a between-unit correlation is more descriptive of a data set. Thus, this paper uses a within and between analysis (WABA) technique (cf. Dansereau, Alutto, Markham, \& Dumas, 1982) that makes no a priori assumption about which source of variance for a multiple-data base is more important. As an alternative to a combined ANCOVA/multiple regression procedure, the WABA technique uses weighted unit averages in calculating between-unit correlations as well as correlations based on within-unit variation. In a single step, data are transformed prior to entry into a correlation program by partitioning each respondent's raw score on each measure into two components. These two components are a weighted between-unit score, which is shared by all members of a supervisory unit, and a withinunit component, which is that individual's unique, relative position above or below the units' average (see McNemar, 1955). Thus, supervisory units are equated with statistical cells. The following substantive research question will be addressed in order to illustrate this procedure: To what extent are matched leadership reports from subordinates and supervisors affected by the problem of betweenunit and within-unit variation?

\section{Method}

In using any procedure which focuses upon supervisory groups as the unit of analysis and, therefore, as statistical cells, an experimental design perspective may prove helpful. In a sense, reports from individual respondents can be viewed as validated by the degree to which they are influenced by their group membership. If respondent reports 
are not affected by work group membership, then there will be little variation based on these cells. In this procedure it is necessary to accurately define each supervisory unit and to locate each subordinate in his/her proper group.

\section{耳直e Setting}

In this study, 107 managers were interviewed concerning the leadership style of their superiors. The 107 managers formed the entire managerial hierarchy of the production department of a large metals extraction firm. (This subset of the entire system was taken to minimize the types of roles and the number of management levels in the study. Three levels of management were represented.)

Four procedures were employed to insure that work groups were accurately defined. First, each manager's reporting relationship was obtained from organizational records. Second, structured interviews were scheduled so that managers who were higher in the organization were interviewed before their subordinates. These managers then listed the people who worked for them. Third, their reports were then checked against the information provided by the organization's records. Finally, during the interview, each respondent was asked to verify that the name of his/her superior was correctly listed on the questionnaire.

All 107 managers filled out questionnaires as subordinates and described their relationship with their superiors. After completion of this portion of the interview, 30 of the 107 managers completed a second set of questions about each of their subordinates. In other words, while 107 managers described their 30 supervisors, only 77 respondents completed just one set of instruments as subordinates. The remaining 30 respondents filled out two sets of interview questionnaires: one from a subordinate perspective and one from a superior perspective. In this way mathed superior-subordinate reports were obtained describing 107 different dyadic reporting relationships. This multirater procedure for assessing matched superior-subordinate reports has been used in previous studies of leadership (see Dansereau, Graen, \& Haga, 1975).

\section{Meastares}

The results of analyzing three measures are reported here to illustrate the WABA technique and its inference-drawing procedure.

Leadership attention: Amount received. Leadership was measured by the Leadership Attention Scale. This scale (cf. Dansereau et al, 1975) contains nine items designed to assess various forms of personal consideration of the subordinate by the supervisor. Sample items include the following: assurances of confidence from the superior, support and attention for feelings, information about the current and future states of the unit, feedback from the superior to the subordinate about job performance, and so forth. (For a complete listing, see Markham, 1978.) Subordinates responded to these questions in terms of their superior's actions, e.g., "How much attention from your supervisor are you getting?' Response categories included almost none, a little, a moderate amount, quite a bit, and a great deal. The coefficient alpha for this scale was .92 . The mean was 31.38 with a standard deviation of 6.38 .

Leadership attention: Amount given. Each of the nine items above was also asked of those 30 managers who had other managers reporting to them. These managers described each of their subordinates individually. Each question was rephrased so as to reflect a superior's viewpoint. Thus, each superior was asked to predict the amount of attention and support that a particular subordinate perceived he/she was getting. This procedure resulted in reports about 107 subordinates. The coefficient alpha for this scale was .89. The mean was 29.11 with a standard deviation of 5.63 .

Subordinate satisfaction with superior. Because many studies of leadership have included various measures of satisfaction (see Stogdill, 1974), this measure was also included to add to the scope of the validity check. The use of a nonleadership measure helps rule out the possibility that any results are solely derived from response bias to similar questions. This scale is composed of six items describing the positive aspects of a supervisor from the perspective of a subordinate. It is a portion of the Role Orientation Index (Graen, Dansereau, \& 
Minami, 1972). This index was adapted, in turn, from the Job Description Index. The coefficient alpha was .84 . The mean was 8.32 with a standard deviation of 2.59 .

\section{Analytical Proced}

The analytical procedure consisted of five steps. First, zero-order correlations based on individuals were computed. (This step corresponds to $r x y, t$ in the Covariance Theorem.) Second, group means were calculated with supervisory groups aligned as cells in a one-way ANOVA. Third, these group averages were used for the computation of weighted unit-average correlations. (This step corresponds to $r x, b$ in the Covariance Theorem.) Fourth, after the effects of the units were removed, the residual cror correlation matrix was computed. (This step corresponds to the last part of the last term of the Covariance Theorem, and it is labeled $r x y, w_{\text {。 }}$ ) Fifth, after assessing levels of significance of these correlations, given the independence of the betweencell and within-cell scores, the between-unit correlations were compared to the within-unit correlations to infer the appropriate unit of analysis. A version of the $\mathbb{Z}$ test was used for this last inferential step.

\section{Pesults}

The results for the first two steps of this analytical procedure are shown in Table 1 . This table includes the measures' means, standard deviations, and the $\mathbb{R}^{2}$ and $F$ for the one-way ANOVA that was used to partial out supervisory group differences.

The results of the third and fourth steps of the procedure are shown in Table 2. This table includes the raw individual correlations, the weighted between-unit correlations, and the residual withinunit correlations. For each of the relationships among the measures, three component correlations are displayed. The zero-order, total individual correlations are shown above the line for each relationship. The degrees of freedom for any total correlation are based upon 107 individuals. For each relationship, the two component correlations are shown below the line. The first correlation, shown on the left, is based on weighted group average scores. The degrees of freedom for this correlation are dependent on the number of supervisory units, and in this case $J=30$. The second correlation, shown on the right, is the residual, within-unit correlation after the differences between supervisory units have been partialled out. The degrees of freedom for this correlation are based on the residual term $N-J$.

\section{Leadership Convergence}

The total individual correlation between the superiors' reports and the subordinates' reports describing the amount of leadership attention (Table 2) was $r=.15(n . s)$. Given this result, a researcher might conclude that there is no convergent

Table 1

WABA Computational Procedure for Matched Superior Subordinate Correlated Reports

\begin{tabular}{|c|c|c|c|c|c|}
\hline \multirow[b]{2}{*}{ Measure } & \multirow[b]{2}{*}{ Mean } & \multirow[b]{2}{*}{ S.D. } & \multicolumn{2}{|c|}{ Between-Unit } & \multirow{2}{*}{$\frac{(\mathrm{ANOVA})}{\mathrm{R}^{2}}$} \\
\hline & & & $\mathrm{F}$ & $p^{<}$ & \\
\hline $\begin{array}{l}\text { 1. Leadership Attention Given } \\
\text { (Superior Report) }\end{array}$ & 31.3 & 6.38 & $5.67 * x$ & .001 & $68 \%$ \\
\hline $\begin{array}{l}\text { 2. Leadership Attention Received } \\
\text { (Subordinate Report) }\end{array}$ & 29.1 & 5.63 & 1.59 & .054 & $38 \%$ \\
\hline $\begin{array}{l}\text { 3. Satisfaction with Supervisor } \\
\text { (Subordinate Report) }\end{array}$ & 8.3 & 2.59 & 1.61 & .051 & $38 \%$ \\
\hline
\end{tabular}

* $\mathrm{p}<.05$; * $\mathrm{p}<.01$.

Note: The degrees of freedom for the zero-order, total individual correlations are based on $\mathrm{N}=107$ individuals, those for the between-unit correlations on $J=30$ units, and those for the residual within-unit correlations on $\mathbb{N}-J=77$. 
validity. In traditional leadership research, however, the component of variation of theoretical interest is based on supervisory units, not raw individual scores (Seeman, 1957; Sheridan \& Vredenburg, 1979). For superiors' reports of leadership attention, a theoretical focus on groups seems plausible because $68 \%$ of the variation in these supervisory reports was attributable to differences between the 30 supervisory groups. Furthemore, $38 \%$ of the variance in subordinates' reports occurred between groups for both the second and third scale. These findings might result in an inference that the phenomenon being investigated is a betweenunit phenomenon because a significant amount of variance occurs at that level of analysis.

Before such an inference can be drawn, however, there must be significant covariation between superior and subordinate leadership descriptions at the supervisory level of analysis. Because the correlation between the amount of leadership attention given and the amount received based on unit averages is $r_{\mathrm{b}}=-.12\left(n . s_{.}\right)$, the data do not support such an inference. Contrary to traditional expectations, however, the within-unit correlation between the two leadership reports was significant with $r_{w}=.46$. Thus, these data appear to describe a situation in which there is convergence between superiors and subordinates when describing individuals who receive relatively higher amounts of leadership attention when compared to just the members of their own wnit. Even though there was a fair amount of variation between supervisory units for both variables, significant covartation did not occur based upon differences between groups.

When the superiors" reports of leadership attention given are compared with subordinates' reports of satisfaction with their supervisor, similar results are obtained. The total individual correlation was $r=.23$. Removing the between-unit variation resulted in a within-unit correlation of $r_{w}=.39$. The between-unit correlation was $r_{\mathrm{b}}=.11$ ( $($ p.s.). Again, the statistical imposition of supervisory units appeared to result in a nontraditional conclusion.

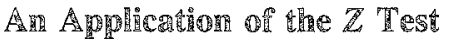

Because the WABA technique forces the simultaneous consideration of two scores, a proce- dure is needed to test the significance of the difference between these two component correlations. Dansereau et al. (1982) have recommended the use of a standard $\mathbb{Z}$ test. Since the between- and withinunit correlations are statistically independent (McNemar, 1955), it is possible to test the difference between them using the standard $r$ to $\mathbb{Z}$ formula for testing the difference betweer independent correlations (Guilford, 1965). Whem neithen component correlation can be considered error, the equation can be written as

$$
Z=\frac{Z^{B}-Z^{B} W}{\sqrt{\frac{1}{J-3}+\frac{1}{N-J-3}}}
$$

where $Z^{\prime} b$ is the transformed between-unit correlation and $Z^{\prime} W$ is the transformed within-unit correlation. If $Z^{\prime} b$ or $Z^{\prime} w$ is considered error, a more general form of this equation is

$$
Z=\frac{Z^{8} \text { obs }-Z^{\prime} \text { error }}{\sqrt{\frac{1}{d f_{\text {obs }}-3}}}
$$

where $Z^{\prime}$ obs is either transformed between- or withinunit scores. In this case, these $\mathbb{Z}$ tests for the withinunit correlations have $N-J-3$ degrees of freedom and the between-unit correlations have $J-3$ degrees of freedom. Thus, the denominator in Equation 3 is readily obtained.

Table 3 shows the results of applying Equations 2 and 3 to the set of between- and within-unit correlations from Table 1 . For each pair of correlations, three $\mathbb{Z}$ scores are shown. The $\mathbb{Z}$ scores shown in the first and second columns of the table are based upon Equation 3 . The results in the first column assume that the between-unit correlation is error. The second column's results also ise Equation 3 and assume that the within-unit correlation is error. The last $\mathbb{Z}$ score shown in the last column, based on Equation 2, is a more conservative test and is used when there is no previous knowledge or expectation that either component correlation is error. 

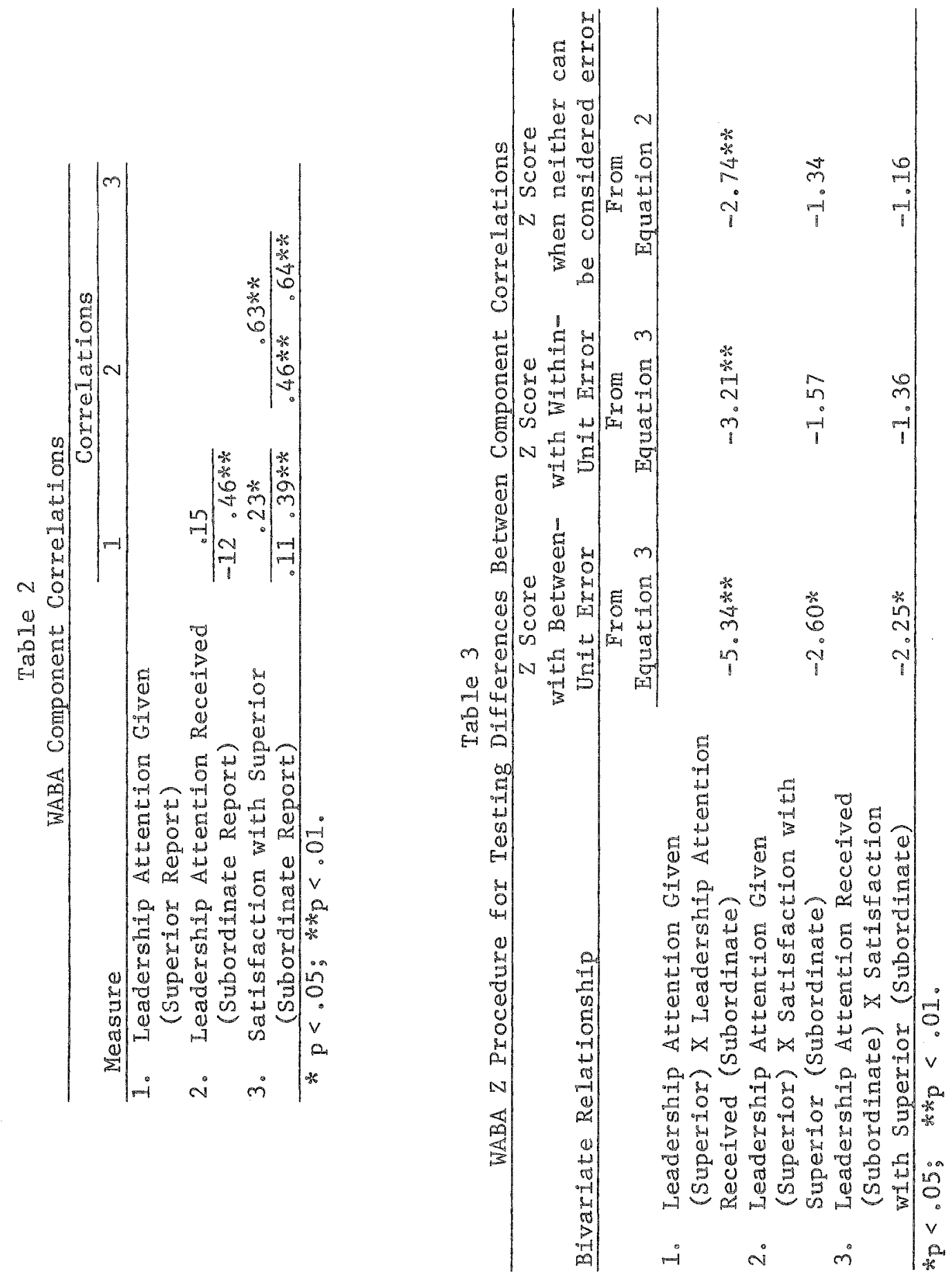
Regardless of which assumption is made, all three $Z$ scores indicate that there are significant differences between the component correlations for the superiors' and subordinates' leadership attention reports. Thus, the within-unit correlations are stronger than the between-unit correlations. A withinunit model is inferred.

As shown in the first column of Table 3 , the difference between the within- and between-unit correlations of the superiors' reports of leadership attention and the subordinates' reports of satisfaction is statistically significant only when an assumption is made that withinumit variation is not error. As shown in the second and third columns, if this assumption is not made (and appropriate degrees of freedom are used in the denominator of the $\mathbb{Z}$ tests), the differences between the two component correlations are not statistically significant.

The difference between the component correlations for the subordinates' descriptions of the amount of leadership attention they receive and their level of satisfaction follows the same pattern as that of the correlations based on superiors' leadership reports and subordinates' satisfaction reports. There is, however, a difference between these two cases based on the results presented in Table 2. In the case of the relationship between raters, the between-cell $r$ was near zero. For the selfreports, the between-cell $r$ was greater than zero.

Before considering the problem posed by these self-reports, a summary of the implications of the $Z$ test may be helpful. The $Z$ test, a two-step procedure, is used to discriminate between the between-unit and within-unit correlations. First, component correlations must be tested for their level of statistical significance. Second, the difference between the component correlations must also be tested. When both steps have been accomplished, an inference is drawn by ruling out the various alternatives. These altematives can be listed as follows:

1. A between-unit condition exists when $r_{\mathrm{b}}$ is significant, $r_{\mathrm{w}}$ is null, and the difference between them is significant;

2. A within-unit condition exists when $r_{w}$ is significant, $r_{\mathrm{b}}$ is null, and the difference between them is significant;
3. An ambiguous condition exists when both $r_{b}$ and $r_{w}$ are significant regardless of the difference between them; and

4. The traditional null condition exists when neither $r_{\mathrm{b}}$ nor $r_{\mathrm{w}}$ is significant regardless of the difference between them (see Dansereau et al., 1981, for a more detailed description).

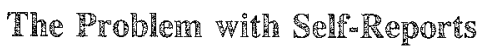

The correlations based only on subordinate selfreports in Table 2 pose an interesting problem of interpretation because they fall in the third category described above. In this case, the subordinates" reports of the amount of leadership attention received are correlated with their reports of satisfaction with their supervisor. The total individual correlation of $r=.63$ was significant. Both component correlations were also significant, thereby resulting in an ambiguous inference as indicated by the third category above. Although it would be tempting to conclude that both between-unit and within-unit phenomena are occurring, there are at least three reasons not to draw this inference.

One possible explanation is that some supervisory units are homogeneous with respect to leadership attention, while others have a large amount of variation. If this were the case, then to make sense of the data one approach would be to define an appropriate structural boundary condition which divides the population of supervisory units into two categories. In one hypothetical subsystem, data might be configured such that an $r_{b}=.46$ and $r_{w}=.00$. In a second subsystem, $r_{\mathrm{b}}$ might be .00 and $r_{\mathrm{w}}=.64$. In this example, by inadvertenty combining these hypothetical subsystems, an ambiguous condition could result. If this were true for the actual data reported here, it would be inappropriate to infer that both a between-unit phenomenon and a withinunic phenomenon are occurring in all of the supervisory groups.

A second reason for assigning this result to an ambiguous condition has to do with parsimony. If each supervisory group is differentiated, and if there is also differentiation between groups, then the use of supervisory groups as statistical cells has not contributed any new knowledge. Thus, whatever 
leadership processes are occurring are perhaps better understood as an individualized situation which is independent of supervisory units. Rather than conclude that both between-unit and within-unit phenomena are occurring, it might be more parsimonious to suggest that neither is supported.

The assumption underlying these two arguments is that both component correlations of the subordinates' self-reports in Table 2 are valid. This assumption also yields a third reason for interpreting these results as indicating an ambiguous condition. Specifically, Eden and Leviatan (1975) have argued that implicit theories of leadership may account for some of the self-reported data in organizational research. Thus, statistically significant correlations from self-reported data might not be valid if they suffer from response bias and have no correspondence to external referents.

The results in Table 2 for subordinate selfreports could be interpreted in this manner. For the leadership-satisfaction relationship, both betweenand within-cell correlations are significantly different from zero. However, when the superiors' reports of leadership attention are compared with the subordinates' report of satisfaction, the betweenunit correlation is no longer significant $\left(r_{b}=.11\right.$, n.s.). Furthermore, the convergence correlation for validating the leadership reports seems to operate only at the within-unit level of analysis. Thus, it is possible that the between-unit correlation for the subordinates' self-reports, while statistically significant, may not be accurate because it cannot be confirmed from the superiors' perspective. In conclusion, because any of these three possibilities cannot be ruled out on the basis of the data provided, these correlated self-reports will not be interpreted as representing both between- and withinunit phenomena.

\section{Discenssion}

The research question posed earlier can now be tentatively answered. The unit-of-analysis problem as represented by between-group and within-group sources of variation has a major effect upon matched supervisor-subordinate leadership convergence correlations. If just the raw correlation based on the total number of individuals were used, a null inference would be drawn from the data $(r=.15$, n.s., from Table 2). Despite the fact that a large amount of variation is accounted for by differences between units, these differences did not translate into systematic covariation at the unit level for these leadership descriptions $\left(r_{\mathrm{b}}=-.12, n . s_{0}\right)$. The imposition of supervisory groups as statistical cells produced a residual correlation $\left(r_{\mathrm{w}}=.46\right)$ that was significantly different from zero and significantly different from its between-unit counterpart. After the application of the $\mathbb{Z}$ test, an inference was drawn that there appears to be valid convergence between superiors and subordinates concerning the amount of leadership attention when a within-unit perspective is used. In traditional leadership research, this within-unit portion of variance is usually considered error (Seeman, 1957).

The results from applying the WABA procedure also demonstrate that a leadership-satisfaction linkage might well fall into the same within-unit condition, depending on which a priori assumption is made. However, the information derived from subordinate self-reports in this study is ambiguous and could not be interpreted. A distinct possibility exists that either one of the component correlations of the subordinate self-reports was not valid even though found to be statistically significant.

The discovery of significant within-unit correlations is contrary to the expectations of many statisticians (see Blalock, 1979). They have argued that the use of correlations based on group averages will be larger than the correlation based on the total number of individuals. The data presented here demonstrate that this is not always true.

The WABA procedure itself has direct implications for measurement in organizational research. If a measure is believed to be a "group" construct, then empirical analyses must be viewed with concern when they do not indicate both that between-unit covariance for two variables is significant and that within-unit covariance is random. Likewise, if a measure is believed to be a "dyadic" construct, then the within-unit covariation should be significant and the between-unit covariance should be essentially random. This approach to the unitof-analysis problem is not restricted to the lead- 
ership area. Sirotnik (1980) has applied it to the issue of organizational climate in educational systems. Theoretically, any issue involving variables such as satisfaction, participation, commitment, and absenteeism is subject to these concerns if the data base has multiple units of analysis that are clearly identified.

The WABA technique has some advantages over the ANCOVA/regression technique. Both are forms of the General Linear Model and therefore result in identical values for within-unit correlations. Between-unit correlations are similar except that WABA makes an adjustment for the size of the unit. For example, in the calculation of the between-unit correlations, WABA makes an adjustment to the unit means by weighting each by the size of the unit. (If unweighted unit averages are entered into a standard least squares regression model, no adjustment is made for the size of each unit.) This is not a problem if all units are the same size. In this data set, some units were larger than others, however, and in such a case, ordinary least squares is not the most efficient technique (Hamnan \& Bumstein, 1974, p. 378). When cells sizes are very different, this problem becomes more pronounced. An additional comparative advantage of WABA is that all results can be displayed in a single table. More important, in WABA an attempt is made to clarify research assumptions about the inference-drawing process through the use of a modified $\mathbb{Z}$ test.

In general terms, WABA falls into a broad category of recently described techniques which are concerned with separating individual from ${ }^{6} \mathrm{con}-$ textual" or group effects. (Firebaugh, 1978; Hannan \& Burstein, 1974; James, Demaree, \& Hater, 1980; Kraemer, 1978; Lincoln \& Zeitz, 1980). The WABA technique is similar to the ANCOVA model proposed by Lincoln and Zeitz (1980) with the exception that the WABA technique places equal emphasis on interpreting the within-unit effects aside from the between-unit effects. Because the Lincoln and Zeitz (1980, p. 396) technique is primarily concerned with the between-unit regression line and its decomposition into a predicted component and a residual component for the purpose of testing the between-unit effect, it can be categorized in the macro end of the aggregation spectrum. On the other end of the spectrum, the James et al. (1980, p. 354) technique is oriented more toward the interpretation of individual effects by examining "the total possible variation in the person variable that is associated with between-group differences."

The WABA technique attempts to establish a middle ground in this micro-macro aggregation issue without a priori assumptions about the appropriate unit of analysis. The ability to empirically test the assumptions conceming the appropriate unit of analysis of a construct or a relationship is a key feature of the WABA procedure. Thus, the $W A B A$ technique can be viewed as an altemative to the theoretical decision-making rule proposed by Firebaugh (1978) for situations when aggregated data are used to provide estimates of individual level relationship without committing the ecological fallacy described by Robinson (1950). Essentially, with WABA there is no need for a cross-level inference from aggregate data to individual level theory or vice versa because all units of analysis are present in the multiple data base, and are matched and tested for the appropriate statistical unit.

\section{祸eferences}

Blalock, H. M. The presidential address: Measurement and conceptualization problems: The major obstacle to integrating theory and research, Americare Sociological Review, 1979, 44, 881-894.

Dansereau, F., Alutto, J., Markham, S., \& Dumas, M. Multiplexed supervision and leadership: An application of within and between analysis. In J. Hunt, $U$. Sekaran, \& C. Schriesheim (Eds.), Leadership: Beyond establishment views. Carbondale IL: Southern Illinois University Press, 1982.

Dansereau, F., \& Dumas, M. Pratfalls and pitfalls in drawing inferences about leader behavior in organizations. In J. Hunt and L. L. Larson (Eds.), Leadership: The cutting edge. Carbondale L: Southern Illinois University Press, 1977.

Dansereau, $\mathbb{F}$., Graen, G., \& Haga, W. A vertical dyad linkage approach to leadership within formal organizations: A longitudinal investigation of the role making process. Orgarizational Behavior and Human Performance, 1975, 13, 46-78.

Eden, D., \& Leviatan, U. Implicit leadership theory as a determinant of factor structure underlying supervisory behavior scales. Journal of Applied Psychology, $1975,60,736-741$. 
Firebaugh, G. A rule for inferring individual-level relationships from aggregate data. American Sociological Review, 1978, 43, 557-572.

Graen, G., Dansereau, F., \& Minami, T. Dysfunctional leadership styles. Organizational Behavior and Human Performance. 1972, 7, 216-236.

Guilford, J. P. Fundamental statistics in psychology and education. New York: McGraw-Hill, 1965.

Hannan, M. T. \& Burstein, L. Estimation from grouped observations. American Sociological Review, 1974, $39,374-392$.

James, L. R., DeMaree, R. G., \& Hater, J. J. A statistical rationale for relating situational variables and individual differences. Organization Behavior and Human Performance, 1980, 25, 354-364.

Katerberg, $\mathbb{R}$. \& Hom, $\mathbb{P}$. The effects of within-group and between-groups variation in leadership. Journal of Applied Psychology, 1981, 66, 218-223.

Kimberly, J. R. Data aggregation in organization research: The temporal dimension. Organization Studies, 1980, 1, 367-377.

Knapp, T. R. The unit-of-analysis problem in applications of simple correlation analysis in educational research. Joumal of Educational Statistics, 1977, 2, $171-186$.

Kraemer, H. C. Individual and ecological correlation in a general context. Behavior Science, 1978, 23, 6772 .

Lincoln, J., \& Zeitz, G. Organizational properties from aggregate data: Separating individual and structural effects. American Sociological Review, 1980, 45, 391408.

McNemar, Q. Psychological statistics. New York: Wiley, 1955.

Markham, S. Leadership and motivation: An empirical examination of exchange and its outcomes. Unpublished doctoral dissertation, State University of New York at Buffalo, 1978.
Przeworski, A., \& Teune, H. The logic of comparative social inquiry. New York: Wiley \& Sons, 1970.

Robinson, W. S. Ecological correlations and the behavior of individuals. American Sociological Review, 1950, $15,351-357$.

Schriesheim, C. A., House, R., \& Kerr, S. Leader initiating structure: A reconciliation of discrepant research results and some empirical tests. Organization Behavior and Human Performance, 1976, 15, 297321.

Seeman, M. A comparison of general and specific leader behavior descriptions. In R. M. Stogdill \& A. E. Coons (Eds.), Leader behavior: Its descriptions and measurement. Columbus: Ohio State University, Burcau of Business Research, 1957.

Sheridan, J. E., \& Vredenburg, D. J. A structural model of leadership influence in a hospital organization. Academy of Management Journal, 1979, 22, 6-21.

Sirotnik, K. A. Psychometric implications of the unitof-analysis problem (with examples from the measurement of organizational climate). Journal of $E d$ ucational Measurement, 1980, 17, 245-282.

Stogdill, R. M. Handbook of leadership: A survey of theory and research. New York: The Free Press, 1974.

\section{Ack}

The authors acknowledge the assistance and support of the Virginia Tech Productivity Research Center and the State University of New York at Buffalo's Research Foundation.

\section{A}

Send request for reprints or further information to Steven E. Markham, Pamplin Hall/Management, Virginia Polytechnic Institute and State University, Blacksburg VA 24061, U.S.A. 